\title{
Lower Levels of Circulating Adiponectin in Elderly Patients with Metabolic Inflammatory Syndrome: A Cross-Sectional Study
}

This article was published in the following Dove Press journal: Diabetes, Metabolic Syndrome and Obesity: Targets and Therapy

\author{
Lina $\mathrm{Ma} \mathbb{D}^{1, *}$ \\ Yanhong $X u^{1,2, *}$ \\ Yaxin Zhang' \\ Tong $\mathrm{Ji}^{1}$ \\ Yun $\mathrm{Li}^{\prime}$
}

\begin{abstract}
'Department of Geriatrics, Xuanwu Hospital, Capital Medical University, National Clinical Research Center for Geriatric Diseases, Beijing 100053 , People's Republic of China; ${ }^{2}$ Department of Geriatrics, Miyun Teaching Hospital of Capital Medical University, Beijing 101500, People's Republic of China
\end{abstract}

*These authors contributed equally to this work
Correspondence: Lina Ma

Department of Geriatrics, Xuanwu Hospital, Capital Medical University, National Clinical Research Center for Geriatric Diseases, \#45 Changchun Street, Xicheng District, Beijing 100053, People's Republic of China Email malina0883@I26.com
Background: Metabolic inflammation plays an important role in the pathogenesis of atherosclerosis, type 2 diabetes, non-alcoholic fatty liver disease, and obesity. The above metabolic disorders often coexist and are closely related with systemic low-grade inflammation. Metabolic inflammatory syndrome (MIS), a cluster of these four metabolic disorders, is a novel concept that is thought to be a better predictor of coronary heart disease than metabolic syndrome. Adiponectin is an adipokine that increases insulin sensitivity and antioxidation as well as has an anti-atherosclerosis effect. It is unclear whether adiponectin is associated with MIS. The objective of this study was to assess whether circulating adiponectin can be used as an indicator for MIS in elderly adults.

Methods: A total of 136 elderly inpatients were divided into two groups: the MIS group and the non-MIS group. Characteristics, inflammatory markers, blood lipid metabolism, insulin resistance, and circulating adiponectin levels were assessed and compared between the two groups. Logistic regression analysis was used to explore the association between the associated factors and MIS.

Results: MIS patients were more likely to have a higher body mass index, have a higher fasting blood glucose level, be smokers, have fundus arteriosclerosis, have lower levels of adiponectin $(4044.90 \pm 3906.82 \mathrm{ng} / \mathrm{mL}$ vs $8503.89 \pm 3523.89 \mathrm{ng} / \mathrm{mL}, p<0.001)$, and have higher levels of inflammation (white blood cell and C-reactive protein) than non-MIS patients. Multiple factor analysis showed that lower adiponectin levels $(\mathrm{OR}=22.937, p<$ 0.001 ) were independently associated with MIS. Adiponectin levels were lower in the vascular disease $(2673.29 \pm 1930.05 \mathrm{ng} / \mathrm{mL}$ vs $7194.38 \pm 4615.83 \mathrm{ng} / \mathrm{mL}, p=0.001)$, atherosclerosis $(2374.14 \quad[1496.41-4263.90] \quad \mathrm{ng} / \mathrm{mL} \quad$ vs $6037.48 \quad$ [3865.61-10,373.60] $\mathrm{ng} / \mathrm{mL}, p<0.001)$, and type 2 diabetes $(2391.73$ [1386.41-4069.68] ng/mL vs 8916.03 [5801.14-12,724.45] ng/mL, $p<0.001$ ) subgroups than in the non-MIS group.

Conclusion: Lower adiponectin levels in serum are associated with MIS. Low adiponectin may be used as a biomarker for atherosclerosis in patients with MIS.

Keywords: metabolic inflammatory syndrome, adiponectin, atherosclerosis, type 2 diabetes, obesity, inflammation, elderly patients

\section{Background}

With an aging population, coronary heart disease has become a serious threat to human health. Metabolic syndrome (MetS) is an established cardiovascular risk factor and has been used to assess cardiovascular and mortality risk in multiple populations. ${ }^{1}$ To date, the pathophysiological mechanism of MetS is still unclear. Central obesity is prevalent in MetS and is highly associated with type 2 diabetes 
(T2D) and cardiovascular disease. Studies have demonstrated that obesity, insulin resistance, and T2D are inextricably linked to macrovascular disease and a systemic low-grade inflammatory response. ${ }^{2}$ First proposed in 2006, the concept of metabolic inflammation (Metflammation) mainly refers to the inflammatory process triggered by excess nutrients and metabolism; its molecular mechanism and signaling pathway are similar to those of traditional inflammatory reactions, but it involves chronic, low-grade inflammation. ${ }^{3}$ The high prevalence of MetS is closely related to changes in living habits, genetic factors, and age. Eating more and exercising less lead to an excess of nutrients and metabolism; this stimulates inflammatory processes, which result in the secretion of inflammatory factors.

Metabolic inflammatory syndrome (MIS) consists of a cluster of metabolic diseases (obesity, non-alcoholic fatty liver disease [NAFLD], T2D, and atherosclerosis), and macrophages play an important role in its manifestation. ${ }^{4-6}$ Patients with two or more of the metabolic disorders listed above could be diagnosed with MIS. In patients with $\mathrm{T} 2 \mathrm{D}$, MIS is a better predictor of coronary heart disease than MetS; ${ }^{7}$ thus, MIS could have better clinical applications than MetS in improving early screening, prevention, and research of metabolic diseases.

Adiponectin is an adipocyte-derived cytokine that is closely associated with obesity, altered body fat distribution, insulin resistance, and atherosclerosis. It plays an important role in maintaining metabolic homeostasis. ${ }^{8}$ The relationship between adiponectin and cardiovascular disease has been studied over the past two decades. ${ }^{9}$ Research shows that adiponectin is an independent predictor of aortic atherosclerosis in T2D patients. ${ }^{10}$ Thus, it is considered to be an anti-inflammatory and antiatherogenic protein. However, serum adiponectin levels in MIS remain unknown. Considering the high prevalence of metabolic diseases in older adults, we aimed to examine the relationship between circulating adiponectin and atherosclerosis in older adults with MIS.

\section{Methods}

\section{Participants}

A total of 136 patients ( $\geq 60$ years old) from Xuanwu Hospital, Capital Medical University were recruited from July 2014 to April 2015 for this cross-sectional study. The study included patients aged 60 years or more who agreed to participate in the study. Patients with acute heart failure, autoimmune disease, infectious disease, cancer, liver dysfunction, and blood system disease were excluded. Ethics Committee of Xuanwu Hospital Capital Medical University approved this study. All participants signed an informed consent form before the enrollment.

\section{Data Collection}

A questionnaire was administrated to collect information on chronic diseases, family status, economic status, and occupation. Echocardiography, carotid ultrasound, and lower extremity vascular ultrasound data were also collected. Blood samples were collected after overnight fasting. Inflammatory markers, such as white blood cell count and C-reactive protein (CRP) levels, and lipid markers, such as triglycerides (TG), total cholesterol (TC), low density lipoprotein cholesterol (LDL-C), and high-density lipoprotein cholesterol (HDL-C), were tested. The insulin resistance index (IRI) was calculated as follows: IRI= fast plasma glucose $(\mathrm{mmol} / \mathrm{L}) \times$ fast serum insulin $(\mathrm{mIU} / \mathrm{L}) /$ 22.5. Circulating adiponectin was measured using ELISA, following instructions provided by the manufacturer (R\&D Systems, Minneapolis, MN, USA).

\section{MIS Criteria}

Patients with two or more of the following four diagnoses (atherosclerosis, T2D, NAFLD, or obesity) were considered to have MIS. For a diagnosis of atherosclerosis, at least one of the following diagnostic criteria had to be met: a clear history of coronary heart disease or a diagnosis of coronary heart disease using echocardiography, coronary angiography, electrocardiogram, or other diagnostic methods; a history of cerebrovascular disease or a CT- or MRI-based diagnosis of cerebral hemorrhage or cerebral infarction; carotid artery with plaque or stenosis; lower extremity arterial stenosis, atherosclerosis, or occlusion; or the presence of fundus arteriosclerosis. The criteria indicating the presence or absence of cardiovascular disease was determined by reviewing the results of echocardiography, coronary angiography, electrocardiogram, and carotid ultrasound.

\section{Statistical Methods}

EpiData software was used to input data, and secondary input and automatic verification were performed to ensure the accuracy of data entry. All data were processed using the SPSS 11.5 (SPSS Inc., Chicago, IL) or GraphPad Prism 7.0 software (GraphPad Software Inc., CA, USA), Data were presented as mean \pm standard deviation or mean 
rank or number and percentage. The difference in the characteristics between the two groups was evaluated by chi-square test or Fisher's exact probability method for categorical variables, independent $t$-test for continuous variables and Kruskal-Wallis comparisons for the abnormally distributed continuous variables. Stepwise logistic regression was done to explore the association between the various factors as independent variables and MIS as the dependent variable. $p<0.05$ was considered statistically significant.

\section{Results}

The 136 elderly inpatients were divided into two groups: the MIS group $(n=104)$ and the non-MIS group $(n=32)$. MIS patients were more likely to be younger, smokers, and have a higher body mass index than non-MIS patients, whereas there was no difference in waist-hip ratio between the two groups (Table 1). Elderly adults with MIS had higher fasting blood glucose levels and more fundus arteriosclerosis than elderly adults without MIS, while there were no differences in lipid levels, fasting insulin levels, IRI, carotid plaque, or carotid stenosis (Table 1).

The MIS group had a lower level of adiponectin (4044.90 $\pm 3906.82 \mathrm{ng} / \mathrm{mL}$ vs $8503.89 \pm 3523.89 \mathrm{ng} / \mathrm{mL}, p<0.001)$ and higher white blood cell counts $\left(7.04 \pm 2.3210^{9} / \mathrm{L}\right.$ vs $5.81 \pm 1.57$ $\left.10^{9} / \mathrm{L}, p=0.006\right)$ and CRP levels $(2.21[0.91-4.72] \mathrm{mg} / \mathrm{L}$ vs $1.01[0.54-6.70] \mathrm{mg} / \mathrm{L}, p=0.014$ ) than the non-MIS group (Table 1). The results of logistic regression are shown in Table 2. Lower adiponectin $(p<0.001)$, higher fasting glucose levels $(p<0.001)$ and smoking $(p=0.007)$ were independently associated with MIS.

In subgroup analysis, we divided the MIS patients into two subgroups based on the presence or absence of vascular disease. We found lower adiponectin levels in the MIS subgroup with vascular disease than in the MIS subgroup without vascular disease $(7194.38 \pm 4615.83 \mathrm{ng} / \mathrm{mL}$ vs $2673.29 \pm 1930.05 \mathrm{ng} / \mathrm{mL}, p=0.001)$.

Table I Comparison of Characteristics, Lipids, Insulin Resistance and Inflammation Between the Two Groups

\begin{tabular}{|c|c|c|c|c|}
\hline & MIS $(n=104)$ & Non-MIS $(n=32)$ & $\chi^{2 / \mathrm{t}}$ & $p$ \\
\hline \multicolumn{5}{|l|}{ Characteristics } \\
\hline Age $(y)$ & $68.08 \pm 8.80$ & $73.78 \pm 9.82$ & 3.121 & 0.002 \\
\hline Gender (Male, n, \%) & $58(55.8)$ & $15(46.9)$ & 0.779 & 0.378 \\
\hline Smoking (n, \%) & $38(39.2)$ & $3(13.0)$ & 5.644 & 0.018 \\
\hline WHR & $0.96 \pm 0.06$ & $0.93 \pm 0.07$ & 1.900 & 0.060 \\
\hline BMI $\left(\mathrm{kg} / \mathrm{m}^{2}\right)$ & $26.15 \pm 3.36$ & $23.26 \pm 3.1$ & 4.254 & $<0.001$ \\
\hline \multicolumn{5}{|l|}{ Lipids } \\
\hline $\mathrm{TG}(\mathrm{mmol} / \mathrm{L})$ & $1.91 \pm 1.54$ & $1.70 \pm 1.14$ & 0.712 & 0.477 \\
\hline $\mathrm{TC}(\mathrm{mmol} / \mathrm{L})$ & $4.55 \pm 1.45$ & $4.51 \pm 1.22$ & 0.140 & 0.889 \\
\hline LDL-C (mmol/L) & $2.72 \pm 1.23$ & $2.55 \pm 0.89$ & 0.729 & 0.467 \\
\hline HDL-C (mmol/L) & $1.36 \pm 0.40$ & $1.51 \pm 0.46$ & 1.782 & 0.077 \\
\hline \multicolumn{5}{|l|}{ Insulin resistance } \\
\hline Glu (mmol/L) & $7.66 \pm 2.54$ & $5.17 \pm 0.74$ & 8.828 & $<0.001$ \\
\hline FINS (IU/L) & $12.56(7.95-23.52)$ & $11.99(11.13-25.52)$ & 1.328 & 0.059 \\
\hline IRI & $4.14(2.33-7.69)$ & $3.04(2.36-5.39)$ & 1.290 & 0.072 \\
\hline \multicolumn{5}{|l|}{ AS } \\
\hline Carotid plaque (n, \%) & $95(96.0)$ & $24(85.7)$ & 2.340 & 0.126 \\
\hline Carotid stenosis (n, \%) & $26(26.3)$ & $5(18.5)$ & 0.686 & 7.238 \\
\hline Fundus arteriosclerosis (n, \%) & $7 I(8 I .6)$ & $I(20.0)$ & 7.238 & 0.007 \\
\hline \multicolumn{5}{|l|}{ Inflammation } \\
\hline WBC $\left(10^{9} / \mathrm{L}\right)$ & $7.04 \pm 2.32$ & $6.81 \pm 1.57$ & 2.803 & 0.006 \\
\hline $\mathrm{CRP}(\mathrm{mg} / \mathrm{L})$ & $2.21(0.91-4.72)$ & $1.01(0.54-6.70)$ & 1.577 & 0.014 \\
\hline \multicolumn{5}{|l|}{ Adiponectin } \\
\hline Adiponectin (ng/mL) & $4044.90 \pm 3906.82$ & $8503.89 \pm 3523.89$ & 5.772 & $<0.001$ \\
\hline
\end{tabular}

Abbreviations: MIS, metabolic inflammatory syndrome; WHR, waist-hip-ratio; BMI, body mass index; TG, triglycerides; TC, total cholesterol; LDL-C, low density lipoprotein cholesterol; HDL-C, high-density lipoprotein cholesterol; FINS, fast insulin; IRI, insulin resistance index; WBC, white blood cell; CRP, C-reactive protein. 
Table 2 Multivariable Logistic Regression of Factors Associated with MIS

\begin{tabular}{|l|l|l|l|l|l|}
\hline Variables & B & SE & Wald & $\boldsymbol{p}$ & OR (95\% Cl) \\
\hline Smoking & 2.335 & 0.966 & 5.843 & 0.016 & $10.334(1.556-68.653)$ \\
Higher glucose & 3.266 & 1.152 & 8.039 & 0.005 & $26.216(2.741-250.712)$ \\
Lower ADPN & 3.133 & 0.901 & 12.089 & 0.001 & $22.937(3.923-134.120)$ \\
Constant & -7.261 & 1.613 & 20.265 & 0.000 & 0.001 \\
\hline
\end{tabular}

Note: Variables not in the equations were: age $(p=0.294)$, body mass index $(p=0.207)$, white blood cells $(p=0.864)$, and $C$-reactive protein $(p=0.619)$. Abbreviations: MIS, metabolic inflammatory syndrome; ADPN, adiponectin; B, regression coefficient; $\mathrm{SE}$, standard error; Wald, wald statistic; $\mathrm{OR}$, odds ratio; $\mathrm{Cl}$, confidence interval.

Next, we compared adiponectin levels between each of the four diseases that constitute MIS. Among the 104 MIS patients, there were $87(83.7 \%)$ patients with atherosclerosis,
$56(56.7 \%)$ patients with NAFLD, 86 (82.7\%) with T2D, and $29(27.9 \%)$ patients with obesity. Figure 1 shows that adiponectin levels were lower in the MIS subgroups with atherosclerosis (2374.14 [1496.41-4263.90] ng/mL vs 6037.48 [3865.61-10,373.60] ng/mL, $p<0.001)$ and T2D (2391.73 [1386.41-4069.68] ng/mL vs 8916.03 [5801.14-12,724.45] $\mathrm{ng} / \mathrm{mL}, p<0.001)$ than in the non-MIS group.

\section{Discussion}

Adiponectin levels were significantly lower in elderly patients diagnosed with MIS than in elderly patients not diagnosed with MIS. Adiponectin levels were also significantly lower in the vascular disease, atherosclerosis, and T2D subgroups of MIS than in the non-MIS group. This is

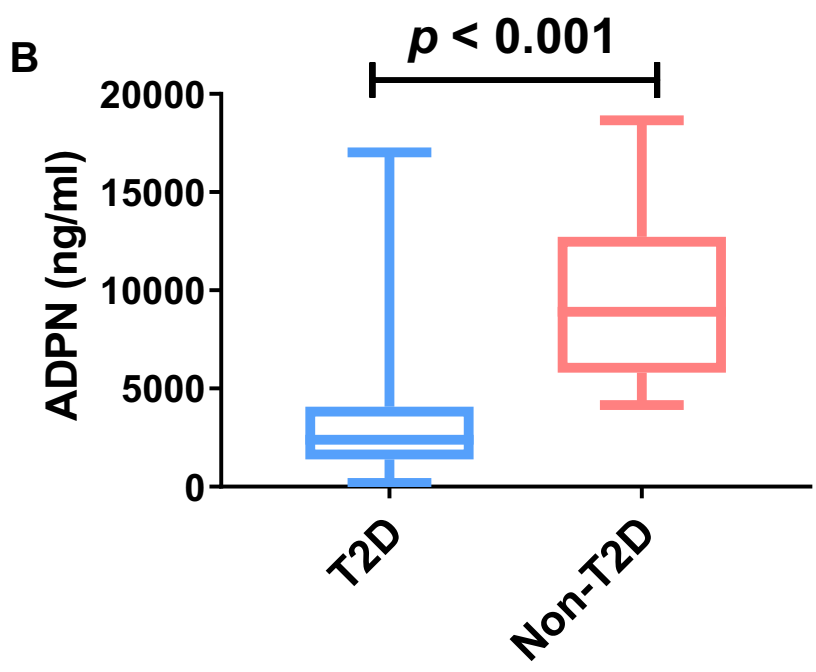

D

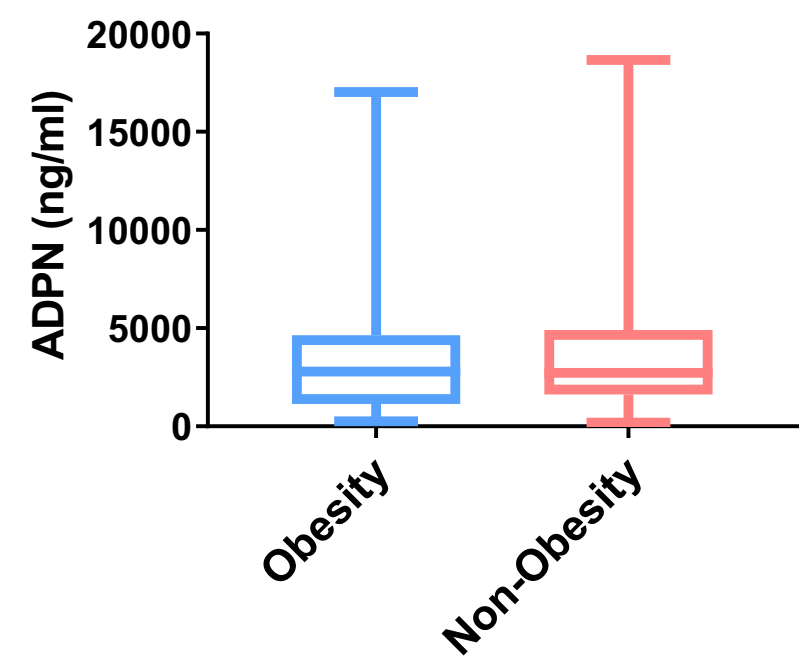

Figure I Comparison of adiponectin levels between each of the four diseases that constitute MIS. Circulating adiponectin levels were compared between AS group and nonAS group (A), T2D group and non-T2D group (B), NAFLD group and non-NAFLD group (C), and obesity group and Non-obesity group (D).

Abbreviations: MIS, metabolic inflammatory syndrome; ADPN, adiponectin; AS, atherosclerosis; T2D, type 2 diabetes; NAFLD, non-alcoholic fatty liver disease. 
consistent with the previous research on the important regulatory role of adiponectin in the development of atherosclerosis. ${ }^{11}$ Adiponectin is one of the most abundant adipokines secreted by fat cells. The human adiponectin gene is located on chromosome 3q27; mutations at this site predispose people to diabetes and cardiovascular disease. ${ }^{12}$ Low adiponectin levels are a risk factor for atherosclerotic disease. Consistent with its role in regulating atherosclerosis, plasma adiponectin levels are associated with atherosclerosis markers, such as inflammation, oxidative stress, and endothelial dysfunction. ${ }^{11}$ Although most studies found low adiponectin levels to be a risk factor for cardiovascular disease, higher adiponectin levels are positively associated with cardiovascular disease in diabetic cohorts with ${ }^{13}$ and without cardiovascular disease. ${ }^{14}$ Other studies found no association between adiponectin and cardiovascular disease. ${ }^{15,16}$ A review unraveled the seemingly paradoxical association between adiponectin and cardiovascular disease; moderate levels of adiponectin exert protective effects on the cardiovascular system while high adiponectin levels indicate advanced cardiovascular disease. ${ }^{17}$ Adiponectin is known to play protective roles in other tissues as well. The role of adiponectin signaling in skeletal muscle has expanded beyond that of a metabolic regulator to include several aspects of skeletal muscle function and maintenance critical to muscle health. ${ }^{18}$

This study showed that patients with MIS have a higher body mass index. Obesity is an inflammatory state. Adipocytes, particularly visceral fat cells, play an important endocrine role in inflammation and dysregulated insulin sensitivity. For excess fat storage, visceral fat cells secrete large amounts of inflammatory cytokines, such as interleukin-6, tumor necrosis factor- $\alpha$, and chemokines. ${ }^{19}$ These chemokines, in turn, promote the migration of macrophages to adipose tissue and greatly increase the release of cytokines. Excessive visceral adipose tissue combined with pro-inflammatory conditions produces systemic inflammation, which increases central obesity.

White blood cell count and CRP levels were elevated in the MIS group compared to those in the non-MIS group, supporting the idea that MIS is a chronic, low-grade inflammatory state. CRP is a non-specific product that is produced in response to infection or tissue damage during the acute phase of inflammation. There is increasing evidence that the inflammatory process plays an important role in the development and progression of atherosclerosis and its complications. ${ }^{20}$ Lipid-rich macrophages, or foam cells, are characteristic of early atherosclerosis.
Macrophage polarization is one of the contributing factors for vascular disease. ${ }^{21}$ Adiponectin inhibits the proliferation of granulocyte-monocytes and inhibits the function of mature macrophages, negatively regulating the immune and inflammatory responses in endothelial cells of the vascular wall. $^{22}$

This study showed that adiponectin levels were reduced in patients with T2D. Adiponectin is negatively correlated with the risk of T2D. ${ }^{23}$ Overnutrition and hyperglycemia can lead to the activation of inflammatory pathways and can increase pro-inflammatory cytokines, such as tumor necrosis factor- $\alpha$ and interleukin -6 , which severely inhibit adiponectin, influencing the development of insulin resistance. ${ }^{24}$ Adiponectin is negatively correlated with fatty acid oxidation, which increases ceramide and diglyceride in plasma and tissues, both of which are believed to inhibit insulin signaling. ${ }^{25}$

This study showed that MIS, specifically atherosclerosis, vascular disease, or T2D, was associated with lower levels of adiponectin, which suggests that a low adiponectin level is a risk factor for coronary heart disease. However, due to the lack of longitudinal data and the small sample size, further studies are needed to confirm these results.

\section{Conclusions}

Low adiponectin levels could potentially be used as a biomarker for the progression of MIS, enhancing early assessment of pathology in patients with coronary heart disease.

\section{Abbreviations}

MIS, metabolic inflammatory syndrome; T2D, type 2 diabetes; NAFLD, non-alcoholic fatty liver disease; MetS, metabolic syndrome; CRP, C-reactive protein; TG, triglycerides; TC, total cholesterol; LDL-C, low density lipoprotein cholesterol; HDL-C, high-density lipoprotein cholesterol; IRI, insulin resistance index.

\section{Ethics Approval and Consent to Participate}

Ethics Committee of Xuanwu Hospital Capital Medical University approved this study. All participants signed an informed consent form.

\section{Data Sharing Statement}

All data published here are under the consent for publication. 


\section{Acknowledgment}

Lina $\mathrm{Ma}$ and Yanhong $\mathrm{Xu}$ are co-first authors for this study.

\section{Author Contributions}

All authors contributed to data analysis, drafting and revising the article, gave final approval of the version to be published, and agree to be accountable for all aspects of the work.

\section{Funding}

This work was supported by the Beijing Natural Science Foundation (7202059), Beijing Municipal Administration of Hospitals Incubating Program (PX2020036), and National Natural Science Foundation of China (81600927).

\section{Disclosure}

The authors report no conflicts of interest in this work.

\section{References}

1. The Global Burden of Metabolic Risk Factors for Chronic Diseases Collaboration. Cardiovascular disease, chronic kidney disease, and diabetes mortality burden of cardiometabolic risk factors from 1980 to 2010: a comparative risk assessment. Lancet Diabetes Endocrinol. 2014;2(8):634-647. doi:10.1016/S2213-8587(14)70102-0

2. Esser N, Legrand-Poels S, Piette J, et al. Inflammation as a link between obesity, metabolic syndrome and type 2 diabetes. Diabetes Res Clin Pract. 2014;105(2):141-150. doi:10.1016/j.diabres.2014.04. 006

3. Hotamisligil GS. Inflammation and metabolic disorders. Nature. 2006;444:860-867. doi:10.1038/nature05485

4. Hu R, Xie Y, Lu B, et al. Metabolic inflammatory syndrome: a novel concept of holistic integrative medicine for management of metabolic diseases. AME Med J. 2018;3:51. doi:10.21037/amj

5. Hu R. Concept and clinical significance of metabolic inflammatory syndrome. Clin Focus. 2016;31(9):960-963.

6. Li R, Hu R. Progress in the pathogenesis of metabolic inflammatory syndrome. Fudan Univ J Med Sci. 2017;44(3):380-384.

7. Hu R, Xie Y, Lu B, et al. High detective rate of "metabolic inflammatory syndrome" in patients with type 2 diabetes. Chin $J$ Endocrinol Metab. 2016;32:27-32.

8. Adiyaman SC, Ozer M, Saydam BO, et al. The role of adiponectin in maintaining metabolic homeostasis. Curr Diabetes Rev. 2019. doi:10.2174/1573399815666190702155733

9. Lara-Castro C, Fu Y, Chung BH, et al. Adiponectin and the metabolic syndrome: mechanisms mediating risk for metabolic and cardiovascular disease. Curr Opin Lipidol. 2007;18:263-270. doi:10.1097/MOL. 0b013e32814a645f
10. Shih CH, Hsu BG, Hou JS, et al. Association of low serum adiponectin levels with aortic arterial stiffness in patients with type 2 diabetes. J Clin Med. 2019;8(6):E887. doi:10.3390/jcm8060887

11. Yanai H, Yoshida H. Beneficial Effects of adiponectin on glucose and lipid metabolism and atherosclerotic progression: mechanisms and perspectives. Int J Mol Sci. 2019;20(5):E1190. doi:10.3390/ijms 20051190

12. Esfahani M, Movahedian A, Baranchi M, et al. Adiponectin: an adipokine with protective features against metabolic syndrome. Iran J Basic Med Sci. 2015;18(5):430-442.

13. Hung WC, Wang CP, Lu LF, et al. Circulating adiponectin level is associated with major adverse cardiovascular events in type 2 diabetic patients with coronary artery disease. Endocr J. 2010;(57):79 3-802. doi:10.1507/endocrj.K10E-020

14. Schottker B, Herder C, Rothenbacher D, et al. Proinflammatory cytokines, adiponectin, and increased risk of primary cardiovascular events in diabetic patients with or without renal dysfunction: results from the ESTHER study. Diabetes Care. 2013;36(6):1703-1711. doi: $10.2337 / \mathrm{dc} 12-1416$

15. Schulze MB, Shai I, Rimm EB, et al. Adiponectin and future coronary heart disease events among men with type 2 diabetes. Diabetes. 2005;54:534-539. doi:10.2337/diabetes.54.2.534

16. Krzyzanowska K, Aso Y, Mittermayer F, et al. High-molecularweight adiponectin does not predict cardiovascular events in patients with type 2 diabetes. Transl Res. 2009;153(4):199-203. doi:10.1016/ j.trsl.2009.01.009

17. Woodward L, Akoumianakis I, Antoniades C. Unravelling the adiponectin paradox: novel roles of adiponectin in the regulation of cardiovascular disease. Br J Pharmacol. 2017;174(22):4007-4020. doi:10. 1111/bph.v174.22

18. Krause MP, Milne KJ, Hawke TJ. Adiponectin-consideration for its role in skeletal muscle health. Int J Mol Sci. 2019;20(7):E1528. doi:10.3390/ijms20071528

19. Tilg H, Moschen AR. Inflammatory mechanisms in the regulation of insulin resistance. Mol Med. 2008;14:222-231. doi:10.2119/200700119.Tilg

20. Nguyen MT, Fernando S, Schwarz N, et al. Inflammation as a therapeutic target in atherosclerosis. J Clin Med. 2019;8(8):E11 09. doi: $10.3390 / \mathrm{jcm} 8081109$

21. Komohara Y, Fujiwara Y, Ohnishi K, et al. Contribution of macrophage polarization to metabolic diseases. $J$ Atheroscler Thromb. 2016;23(1):10-17. doi:10.5551/jat.32359

22. Fang H, Judd RL. Adiponectin regulation and function. Compr Physiol. 2018;8(3):1031-1063.

23. Knights AJ, Funnell AP, Pearson RC, Crossley M, Bell-Anderson $\mathrm{KS}$. Adipokines and insulin action -A sensitive issue. Landes Biosci. 2014;3(4-6):88-96.

24. Hector J, Schwarzloh B, Goehring J, et al. TNF-alpha alters visfatin and adiponectin levels in human fat. Horm Metab Res. 2007;39: 250-255. doi:10.1055/s-2007-973075

25. Maeda N, Shimomura I, Kishida K, et al. Diet-induced insulin resistance in mice lacking adiponectin/ACR P30. Nat Med. 2002;8:73 1-737. doi:10.1038/nm724

\section{Publish your work in this journal}

Diabetes, Metabolic Syndrome and Obesity: Targets and Therapy is an international, peer-reviewed open-access journal committed to the rapid publication of the latest laboratory and clinical findings in the fields of diabetes, metabolic syndrome and obesity research. Original research, review, case reports, hypothesis formation, expert opinion and commentaries are all considered for publication. The manuscript management system is completely online and includes a very quick and fair peer-review system, which is all easy to use. Visit http://www.dovepress.com/testimonials.php to read real quotes from published authors. 